\title{
GASTRONOMÍA TíPICA DE SAMBORONDÓN, COMO PARTE DEL PATRIMONIO INTANGIBLE DE UN PUEBLO.
}

\author{
Julio Fernando Cevallos Ruales, Mgs. \\ Magíster en Docencia Universitaria e Investigación Educativa (Ecuador). \\ Docente Tiempo Completo de la Facultad de Hotelería y Turismo de la Universidad \\ Tecnológica ECOTEC, Ecuador. \\ fcevallos@ecotec.edu.ec
}

\section{ARTÍCULO DE INVESTIGACIÓN}

Recibido: 9 de septiembre de 2019.

Aceptado: 8 de noviembre de 2019.

\section{RESUMEN}

La gastronomía en el mundo ha ido evolucionando con el pasar de los siglos, cada año se encuentran más variedades de deliciosos platos que se pueden degustar en cualquier rincón del planeta, con nuevas variedades, nuevos sabores, nuevas formas. El hombre ha ido experimentando con una gama de ingredientes que ha tenido a la mano, creando recetas únicas y dando un realce al área gastronómica, logrando obtener una aceptación entre las personas que buscan experimentar el placer único de degustar cada bocado de comida, estableciendo platos originarios y reconocidos de su país. Es así, como la gastronomía ha pasado de ser solo un alimento para el arte de la cocina, a convertirse en una pieza fundamental al describir un pueblo, permitiendo conocer sus creencias y forma de vida, mejorando su desarrollo, fomentando su identidad y su diferencia entre una ciudad y otra. En el Ecuador, la gastronomía también ha progresado y, es así, como hay ciudades donde se puede disfrutar de sus diversidades, sus culturas, tradiciones y sus comidas típicas: la región costa (cantón Samborondón). La presente investigación trata de verificar si la gastronomía típica del cantón Samborondón es parte de la identidad del pueblo y si en la actualidad, ésta se está perdiendo.

Palabras clave: gastronomía, tradiciones, ancestrales, típica, ingredientes. 


\section{ABSTRACT}

Gastronomy in the world has evolved over the centuries, every year there are more varieties of delicious dishes that can be tasted in any corner of the planet, with new varieties, new flavors, and new forms. Man has been experimenting with a range of ingredients that he has at hand, creating unique recipes and enhancing the gastronomic area, getting an acceptance among people looking to experience the unique pleasure of tasting in every bite of food, establishing dishes originating and recognized from each country. Thus, as gastronomy has gone from being just a food for the art of cooking, to becoming a fundamental piece when describing a town, allowing to know their beliefs and lifestyle, improving their development, promoting their identity and their difference between one city and another. In Ecuador, gastronomy has also progressed and that's how there are cities where you can enjoy its diversities, its cultures, traditions and its typical foods: the coastal region (canton Samborondón). The present investigation tries to verify if the typical gastronomy of canton Samborondón is part of the identity of the town and if it is currently losing.

Keywords: gastronomy, traditions, ancestral, typical, ingredients.

\section{INTRODUCCIÓN}

Los platos típicos son la representación de la gastronomía propia de un lugar, que hace referencia a las cualidades con las que cuenta, porque según sea la zona en la que se encuentre las variedades de ingredientes y preparaciones hace ver que no en todos los lugares la preparación o el sabor son iguales y que cada territorio demostrará la diferencia que hay en la elaboración y presentación de sus platos, logrando deleitar a quien lo consuma.

Sin embargo, no en todos los lugares es así, las generaciones van cambiando, las tradiciones se van perdiendo, y prefieren la comida rápida por ser más fácil de preparar, a la vez que da buenos ingresos y es más económica, o simplemente ignoran el hecho de poder ofrecer un plato típico porque creen que no cuentan con uno. Otro caso que se evidencia en la actualidad es la fusión de la gastronomía con la ciencia, dando lugar a la gastronomía molecular, considerado como la cocina moderna que va revolucionando a la cocina tradicional. 
Producto de estas reflexiones se plantea la siguiente interrogante: ¿De qué manera influye las diferentes tendencias gastronómicas en la comida típica del cantón Samborondón?, mediante la cual se desarrolla la presente investigación.

\section{REVISIÓN TEÓRICA}

En diferentes estudios se ha analizado la relación entre turismo y gastronomía (Schlüter, 2008; Ascanio, 2009; López, Ribeiro, Orgaz y Marmolejo, 2015; Björk, 2016), lo que refleja la importancia de este tipo de investigaciones. Así, Kivela (2009); Oliveira, 2011), establecen que la gastronomía ocupa un rol importante en la experiencia vivida por el turista y además señalan que algunos viajeros volverían al mismo destino para disfrutar de su gastronomía.

La gastronomía se configura como uno de los elementos estratégicos para la promoción y consolidación de destinos turísticos. Por otra parte, en los últimos años se dinamizan cada vez más los visitantes que viajan motivados por la gastronomía (Oliveira, 2011). En este sentido Flavián (2011) establecen que la gastronomía se puede considerar desde tres perspectivas: como el motivo o experiencia principal de la actividad turística, como una experiencia o motivación secundaria y como una parte de la rutina; descartando la existencia de un solo tipo de tipo de turista gastronómico.

Desde el punto de vista motivacional el turista gastronómico es abordado por Fields (2002). En esta línea, los turistas por lo general están interesados en conocer la cultura local y sus tradiciones, generándose una interacción personal entre turistas y residentes (Bel y Arranz, 2011).

El informe de la OMT (2016) señala que el turista gastronómico participa de las nuevas tendencias del consumo cultural, buscando la autenticidad de los lugares que visita a través de la gastronomía. Así, la cocina tradicional, la calidad de los platos y la hospitalidad, y el servicio son elementos que los turistas valoran de forma positiva dentro de la gastronomía local (Jiménez, López \& González, 2016). De acuerdo con Ignatov (2006) existen distintos tipos de turistas que buscan distintos tipos de experiencias culinarias: los visitantes que solo participan en actividades relacionadas con los alimentos, aquellos que participan en actividades relacionadas con el vino y aquellos 
que participan en ambos, por lo que se deben desarrollar estrategias y productos acordes a cada uno de esos segmentos.

Asimismo, en varios trabajos científicos se identifica el perfil socio-demográfico de los viajeros, las motivaciones y las valoraciones que realizan respecto a determinadas variables relacionadas con el turismo (Brida y Aguirre, 2010; López, Ribeiro, Orgaz y Marmolejo, 2015).

En ese sentido, la satisfacción es uno de los tópicos más abordados en el campo de las investigaciones en turismo (Kozak \& Bigné, 2004). Ésta se define como la valoración que hace el cliente entre el servicio recibido y el servicio esperado (Oliver, 1980). Además, la satisfacción es percibida como un antecedente de la intención de compra (Rust \& Oliver, 1994) y de la lealtad del visitante (Yoon \& Uysal, 2005). Asimismo, es considerada como moderadora de la imagen de un destino (Chi, 2008).

En este marco, Bigné y Font (2000) establecen que si el visitante se va insatisfecho de su visita al lugar, se reduce la probabilidad de repetición y

generará una imagen menos positiva del destino entre sus conocidos. Por otro lado, Mariani y Sorio (2010) destacan la importancia de identificar los elementos que influyen en el nivel de satisfacción de los consumidores de servicios turísticos. En ese contexto, se han realizado varias investigaciones sobre la conducta de los turistas, el valor percibido, la satisfacción y la intención de revisitar (Petrick \& Morais, 2001). Dado que si el turista se va satisfecho con el destino turístico se genera la lealtad del consumidor (Yoon \& Uysal, 2005), Por ello, es necesario analizar la satisfacción respecto a la calidad de los platos típicos de la zona visitantes sobre la gastronomía y las características de la demanda de los establecimientos de restauración.

\section{Descripción del área geográfica}

Ecuador es un país con importantes atractivos turísticos cuyas políticas públicas están encaminadas a mejorar su oferta turística. Los principales mercados emisores de donde provienen los turistas internacionales en el año 2015, los conformaron Colombia, Estados Unidos y Perú. Según datos del MINTUR (2016) a Ecuador en el año 2015 llegaron 1.543,165 turistas internacionales y en el año 2014 llegaron 1'557.006 de turistas internacionales. 
En las exportaciones no petroleras, el turismo ocupa el tercer lugar luego del banano y camarón. De acuerdo al MINTUR (2016) durante el año 2015 el ingreso de divisas por concepto de viajes y transporte de pasajeros alcanzó la suma de 1.557,4 millones de dólares, mientras en el año 2014 recibió 1.487,20 millones de dólares, observando un incremento del 4,7\% con respecto al año anterior, obteniendo un saldo de 532,9 millones en la balanza turística del año 2015.

La provincia de Guayas se encuentra ubicada al occidente de Ecuador con recursos naturales y culturales para ser visitada por muchos turistas nacionales y extranjeros. El cantón Samborondón posee una superficie de 252 kilómetros cuadrados, el cual tiene una forma geométrica de un rectángulo, cuenta con una población de 100.000 habitantes, donde se puede encontrar su cabecera cantonal con el mismo nombre, y sus parroquias son Tarifa en el área rural y La Puntilla en el área urbana, la cual cuenta con 120 recintos.

El cantón Samborondón cuenta con dos estaciones climáticas, una seca y otra lluviosa, las que duran aproximadamente seis meses cada una. La temperatura del cantón oscila entre los 30 y 32 grados centígrados en invierno y de 22 a 25 grados centígrados en época de verano. Su economía se basa principalmente en la agricultura y ganadería, el producto agrícola que más se cosecha es el arroz, también existe la pesca de agua dulce donde se pueden encontrar productos del mar como la corvina, bagre, boca chico y camarón.

En Samborondón se encuentran importantes centros comerciales como: Las Terrazas con cómodos restaurantes, Village Plaza, Río Centro, Plaza Nova, los cuales son ideales para compras y Plaza Lagos Town Center con variedad de restaurantes. Asimismo, existen otros centros comerciales como La Piazza y Bocca donde también se puede degustar de platos de comida nacional o internacional.

Entre los principales atractivos turísticos de Samborondón se destacan el Parque Histórico ubicado en la parroquia La Puntilla, el cual es muy visitado por turistas nacionales y extranjeros, el Malecón de Samborondón, el Parque Acuático Cabecera Cantonal, el Teatro Sánchez Aguilar y el Antiguo Palacio Municipal.

\section{MATERIALES Y MÉTODOS}


Cuestionario y procedimiento

La metodología utilizada para la presente investigación empírica se basó en la realización de un trabajo de campo para recolectar la información en la cual se utilizó un cuestionario elaborado en base a diferentes trabajos (López, Ribeiro, Orgaz \& Marmolejo, 2015; Björk, 2016) donde se encuentran estudios sobre gastronomía y satisfacción.

La presente investigación en lo referente al trabajo de campo se desarrolló entre los meses de enero a febrero de 2019. El cuestionario constaba de preguntas, organizadas técnicamente en secciones. En la primera sección, se obtiene la información sociodemográfica de los visitantes que disfrutaban de la gastronomía. La segunda sección trata sobre el comportamiento de consumo y la tercera sección se refiere a la satisfacción del servicio en los restaurantes de gastronomía típica.

Los visitantes fueron encuestados por estudiantes de la Facultad de Turismo y Hotelería de la Universidad Ecotec, como parte de la recolección de información para su trabajo de Vinculación con la Colectividad, en la parroquia La Puntilla y en la cabecera cantonal, en las áreas externas de los centros comerciales donde se encuentran los restaurantes de comida típica, los cuales llenaron las encuestas de manera independiente y los encuestadores estuvieron muy cerca para explicarles las dudas que podían tener al respecto. Se realizó una prueba piloto con 30 encuestas para validar los cuestionarios y corregirlos. Para pretender obtener los resultados relacionados al estudio se utilizó una mezcla de técnicas de preguntas, para lo cual se utilizaron preguntas cerradas, de opción múltiple y preguntas que servirían para valorar la opinión de los visitantes.

\section{Muestreo y error muestral}

Dado que el tamaño de la población es infinito porque no hay un estudio del número de visitantes que llega al destino, la determinación de la muestra se obtuvo a partir de la ecuación para la estimación de proporciones de este tipo de poblaciones. La variabilidad de la población se estima en un 50\% ( $p=q=0,5)$, valor ampliamente utilizado en investigación social (Burns, 2000).

Se aplicaron 400 cuestionarios, de las cuales 384 fueron válidos, siendo este el tamaño de la muestra, con un margen de error de $+/-5 \%$ y un nivel de confianza del $95 \%$. 
Tabla 1. Indicadores de investigación.

\begin{tabular}{|l|c|}
\hline Población & Visitantes \\
\hline Área geográfica & Cantón Samborondón \\
\hline Período de realización & Enero - marzo 2019 \\
\hline Tipo & Aleatorio simple \\
\hline Nivel de Confianza & $95 \%$ \\
\hline Margen de error & $+/-5 \%$ \\
\hline Validados & 384 \\
\hline
\end{tabular}

Fuente: Elaboración propia a partir del Trabajo de campo.

Los datos recogidos en la presente investigación fueron organizados, tabulados y analizados usando el programa Spss Versión 22. El tratamiento de los datos se ha realizado a través de la utilización de herramientas estadísticas, a continuación, se presentan algunas de las preguntas más relevantes para el respectivo análisis.

\section{RESULTADOS Y DISCUSIÓN}

Tabla 2. Sexo del encuestado.

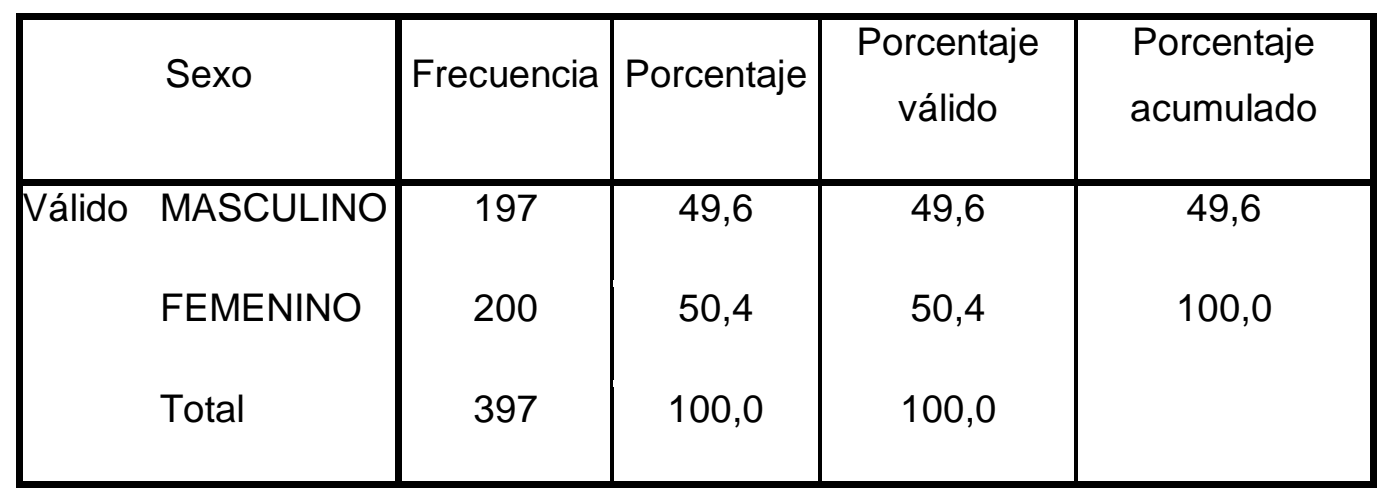

Fuente: Elaboración propia a partir del Trabajo de campo, 2019.

En la tabla 2 se demuestra la homogeneidad de los encuestados el 49.6\%(197) que corresponden al sexo masculino y el $50.4 \%$ (200) al sexo femenino. 
Tabla 3. Sexo del encuestado*Nivel de estudios.

\begin{tabular}{|c|c|c|c|c|c|c|}
\hline \multicolumn{2}{|c|}{} & \multicolumn{4}{|c|}{ NIVEL DE ESTUDIOS } & \multirow{2}{*}{ Total } \\
\cline { 3 - 7 } \multicolumn{2}{|c|}{} & ANALFABETO & PRIMARIA & SECUNDARIA & SUPERIOR & \\
\hline \multirow{2}{*}{$\begin{array}{c}\text { SEXO DEL } \\
\text { ENCUESTADO }\end{array}$} & MASCULINO & 3 & 37 & 108 & 49 & 197 \\
\cline { 2 - 7 } & FEMENINO & 0 & 58 & 47 & 95 & 200 \\
\hline \multicolumn{2}{|c|}{ Total } & 3 & 95 & 155 & 144 & 397 \\
\hline
\end{tabular}

Fuente: Elaboración propia a partir del Trabajo de campo, 2019.

En la tabla 2 se puede observar que los encuestados en relación a su nivel de estudios 3 son personas analfabetas algo que llamó mucho la atención en la zona donde fue recolectada la información, 95 tienen educación primaria 155 educación media, y 144 superior, en la misma tabla se determina que 197 corresponden al género masculino, 3 de ellos son analfabetos, 37 tienen educación primaria, 108 secundaria y 49 superior; 200 al género femenino 58 con educación primaria, 47 educación media y 95 con educación superior.

Tabla 4. Considera si hay pérdida de recetas típicas.

\begin{tabular}{|l|l|c|c|c|c|}
\hline \multicolumn{2}{|c|}{} & \multicolumn{3}{|c|}{$\begin{array}{c}\text { CONSIDERA QUE HAY PERDIDA } \\
\text { DE RECETAS }\end{array}$} \\
\cline { 3 - 5 } \multicolumn{2}{|c|}{} & SI & NO & NS/NR & \multirow{2}{*}{ Total } \\
\hline $\begin{array}{l}\text { SEXO DEL } \\
\text { ENCUESTADO }\end{array}$ & MASCULINO & 176 & 21 & 0 & $\mathbf{1 9 7}$ \\
\cline { 2 - 6 } & FEMENINO & 164 & 17 & 19 & $\mathbf{2 0 0}$ \\
\hline Total & $\mathbf{3 4 0}$ & $\mathbf{3 8}$ & $\mathbf{1 9}$ & $\mathbf{3 9 7}$ \\
\hline
\end{tabular}

Fuente: Elaboración propia a partir del Trabajo de campo, 2019.

En la tabla 4 se demuestra que los encuestados 340 consideran que, existe pérdida de recetas típica en el Cantón Samborondón, 38 manifiestan que no hay pérdida, y 19 de ellos desconocen si hay pérdida o no, se determina que de acuerdo a la información recibida que hay recetas que se han perdido.

Tabla 5. Consulta si ha preparado platos tradicionales.

\begin{tabular}{|l|l|c|c|c|c|}
\hline \multirow{2}{*}{} & \multicolumn{3}{|c|}{$\begin{array}{r}\text { PREPARACIÓN PLATOS } \\
\text { TRADICIONALES }\end{array}$} & \multirow{2}{*}{ Total } \\
\cline { 3 - 6 } & SI & NO & NS/NR & T97 \\
\hline $\begin{array}{l}\text { SEXO DEL } \\
\text { ENCUESTADO }\end{array}$ & MASCULINO & 193 & 2 & 2 & $\mathbf{1 9 7}$ \\
\cline { 2 - 6 } & FEMENINO & 182 & 0 & 18 & $\mathbf{2 0 0}$ \\
\hline Total & $\mathbf{3 7 5}$ & $\mathbf{2}$ & $\mathbf{2 0}$ & $\mathbf{3 9 7}$ \\
\hline
\end{tabular}

Fuente: Elaboración propia a partir del Trabajo de campo, 2019. 
Los encuestados concuerdan tanto hombres como mujeres 375, manifiestan que alguna vez sí han preparado platos tradicionales/ típicos, y solo dos no han preparado plato alguno, lo que se concluye que las recetas tradicionales o típicas se encuentran en la memoria viva de la población.

Tabla 6. Consigue ingredientes para preparar platos típicos.

\begin{tabular}{|c|l|c|c|c|c|}
\hline \multicolumn{2}{|c|}{} & \multicolumn{3}{|c|}{ CONSIGUE INGREDIENTES } & \multirow{2}{*}{ Total } \\
\cline { 3 - 6 } \multicolumn{2}{|c|}{} & SI & NO & NS/NR & 197 \\
\hline $\begin{array}{c}\text { SEXO DEL } \\
\text { ENCUESTADO }\end{array}$ & MASCULINO & 196 & 1 & 0 & $\mathbf{2 0 0}$ \\
\cline { 2 - 5 } & FEMENINO & 182 & 2 & 16 & $\mathbf{3 9 7}$ \\
\hline Total & $\mathbf{3 7 8}$ & $\mathbf{3}$ & $\mathbf{1 6}$ & \\
\hline
\end{tabular}

Fuente: Elaboración propia a partir del Trabajo de campo, 2019.

Se procede a indagar si en la comunidad se ofertan productos con los cuales se preparan los platos típicos 378 declaran que sí, existen los productos, 3 consideran que no, 16 desconocen la temática. Se concluye que la materia prima con la que se preparan los platos si están a disposición de la comunidad, por lo que el problema radica en la comunidad por la no preparación de esos platos.

Tabla 7. Calidad de los platos que se ofertan.

\begin{tabular}{|l|l|c|c|c|c|c|}
\hline \multirow{2}{*}{} & \multicolumn{5}{|c|}{ CALIDAD DE LOS PLATOS } & \\
\cline { 2 - 7 } & & MALO & $\begin{array}{c}\text { BUEN } \\
\text { O }\end{array}$ & $\begin{array}{c}\text { MEDIANAMENT } \\
\text { E BUENO }\end{array}$ & $\begin{array}{c}\text { MUY } \\
\text { BUENO }\end{array}$ & Total \\
\hline $\begin{array}{l}\text { SEXO DEL } \\
\text { ENCUESTAD } \\
\text { O }\end{array}$ & $\begin{array}{l}\text { MASCULIN } \\
\text { O }\end{array}$ & 3 & 37 & 108 & 49 & $\mathbf{1 9 7}$ \\
\cline { 2 - 7 } & FEMENINO & 0 & 58 & 47 & 95 & $\mathbf{2 0 0}$ \\
\hline Total & $\mathbf{3}$ & $\mathbf{9 5}$ & $\mathbf{1 5 5}$ & $\mathbf{1 4 4}$ & $\mathbf{3 9 7}$ \\
\hline
\end{tabular}

Fuente: Elaboración propia a partir del Trabajo de campo, 2019.

Se consulta acerca de la calidad de los platos que los prestadores de servicios que ofertan en los restaurantes del Cantón con el propósito de determinar el nivel de satisfacción de los comensales, obteniendo los siguientes resultados: 3 consideran que es mala, 95 que son buenos, 155 medianamente buenos y 144 que son muy buenos; concluyendo que la preparación de y la calidad de los platos ofertados son aceptables por los usuarios. 
Tabla 8. Prueba no paramétrica de PEARSON.

\begin{tabular}{|c|c|c|c|c|c|c|c|c|c|c|}
\hline \multicolumn{11}{|c|}{ Correlaciones } \\
\hline & & $\begin{array}{c}\text { SEXO DEL } \\
\text { ENCUESTADO }\end{array}$ & $\begin{array}{l}\text { NIVEL DE } \\
\text { ESTUDIOS }\end{array}$ & $\begin{array}{c}\text { RESIDE EN } \\
\text { SALITRE }\end{array}$ & $\begin{array}{l}\text { DENOMINA } \\
\text { COCINA } \\
\text { ANCESTRAL }\end{array}$ & \begin{tabular}{|c|} 
PREPARADO \\
PLATOS \\
TRADICIONAL \\
ES \\
\end{tabular} & \begin{tabular}{|c|} 
CONSIGUE \\
INGREDIENTES
\end{tabular} & \begin{tabular}{|c|} 
LOS \\
UTENSILLOS \\
SONIGUALES \\
O DISTINTOS \\
\end{tabular} & $\begin{array}{l}\text { CONSIDERA } \\
\text { QUE HAY } \\
\text { PERDIDA DE } \\
\text { RECETAS } \\
\end{array}$ & $\begin{array}{l}\text { CALIDAD DE } \\
\text { LOS PLATOS }\end{array}$ \\
\hline \multirow{3}{*}{$\begin{array}{c}\text { SEXO DEL } \\
\text { ENCUESTADO }\end{array}$} & \begin{tabular}{|l} 
Correlación de \\
Pearson \\
\end{tabular} & 1 & 098 & ,314" & ,010 & , 169" & ,206" & ,250* & $168^{* *}$ & 098 \\
\hline & Sig. (bilateral) & & ,051 & ,000 & 841 & ,001 & ,000 & ,000 & 001 & ,051 \\
\hline & $\mathrm{N}$ & 397 & 397 & 397 & 397 & 397 & 397 & 397 & 397 & 397 \\
\hline \multirow{3}{*}{$\begin{array}{l}\text { NIVEL DE } \\
\text { ESTUDIOS }\end{array}$} & $\begin{array}{l}\text { Correlación de } \\
\text { Pearson } \\
\end{array}$ & 098 & 1 & ,042 &,$- 315^{* *}$ &,$- 134^{* *}$ &,- 086 &,- 011 &,$- 136^{\prime \prime}$ & $1,000^{* *}$ \\
\hline & Sig. (bilateral) & ,051 & & ,399 & ,000 & ,007 & ,087 & ,823 & ,007 & 0,000 \\
\hline & $\mathrm{N}$ & 397 & 397 & 397 & 397 & 397 & 397 & 397 & 397 & 397 \\
\hline \multirow{3}{*}{$\begin{array}{l}\text { RESIDE EN } \\
\text { SALITRE }\end{array}$} & \begin{tabular}{|l|}
$\begin{array}{l}\text { Correlación de } \\
\text { Pearson }\end{array}$ \\
\end{tabular} & ,314" & ,042 & 1 &,- 056 & ,094 & ,129" & ,247" & ,008 & 042 \\
\hline & Sig. (bilateral) & ,000 & 399 & & 262 & ,062 & ,010 & ,000 & 881 & 399 \\
\hline & $\mathrm{N}$ & 397 & 397 & 397 & 397 & 397 & 397 & 397 & 397 & 397 \\
\hline \multirow{3}{*}{$\begin{array}{c}\text { DENOMINA } \\
\text { COCINA } \\
\text { ANCESTRAL }\end{array}$} & \begin{tabular}{|l|} 
Correlación de \\
Pearson
\end{tabular} & ,010 &,$- 315^{*}$ &,- 056 & 1 & ,640" &, $510^{* \prime}$ & ,404" & ,386" &,$- 315^{\prime \prime}$ \\
\hline & Sig. (bilateral) & 841 & ,000 & 262 & & ,000 & ,000 & ,000 & ,000 & ,000 \\
\hline & $\mathrm{N}$ & 397 & 397 & 397 & 397 & 397 & 397 & 397 & 397 & 397 \\
\hline \multirow{3}{*}{\begin{tabular}{|c|} 
PREPARADO \\
PLATOS \\
TRADICIONAL \\
ES
\end{tabular}} & \begin{tabular}{|l} 
Correlación de \\
Pearson \\
\end{tabular} & , 169" &,$- 134^{* *}$ & ,094 & ,640" & 1 & ,942" & ,801" & ,728" &,$- 134 "$ \\
\hline & Sig. (bilateral) & 001 & ,007 & ,062 & ,000 & & ,000 & ,000 & ,000 & ,007 \\
\hline & $\mathrm{N}$ & 397 & 397 & 397 & 397 & 397 & 397 & 397 & 397 & 397 \\
\hline \multirow{3}{*}{$\begin{array}{l}\text { CONSIGUE } \\
\text { INGREDIENTES }\end{array}$} & \begin{tabular}{|l|}
$\begin{array}{l}\text { Correlación de } \\
\text { Pearson }\end{array}$ \\
\end{tabular} & ,206" &,- 086 & ,129" &, $510^{* \prime}$ & ,942" & 1 & ,894" & ,769" &,- 086 \\
\hline & Sig. (bilateral) &, 000 & 087 & , 010 & ,000 &, 000 & & ,000 & ,000 & ,087 \\
\hline & $\mathrm{N}$ & 397 & 397 & 397 & 397 & 397 & 397 & 397 & 397 & 397 \\
\hline \multirow{3}{*}{\begin{tabular}{c|} 
LOS \\
UTENSILLOS \\
SONIGUALES \\
O DISTINTOS
\end{tabular}} & \begin{tabular}{|l} 
Correlación de \\
Pearson \\
\end{tabular} & ,250" &,- 011 & 247" & ,404" & $801^{*}$ & 894 & 1 & ,689" &,- 011 \\
\hline & Sig. (bilateral) & ,000 & 823 & ,000 & ,000 & ,000 & ,000 & & ,000 & ,823 \\
\hline & $\mathrm{N}$ & 397 & 397 & 397 & 397 & 397 & 397 & 397 & 397 & 397 \\
\hline \multirow{3}{*}{\begin{tabular}{c|} 
CONSIDERA \\
QUE HAY \\
PERDIDA DE \\
RECETAS \\
\end{tabular}} & \begin{tabular}{|l|}
$\begin{array}{l}\text { Correlación de } \\
\text { Pearson }\end{array}$ \\
\end{tabular} & , 168" &,$- 136^{* *}$ & ,008 & ,386" & ,728" & ,769" & ,689" & 1 &,$- 136^{\prime \prime}$ \\
\hline & Sig. (bilateral) & ,001 & ,007 & 881 & ,000 & ,000 & ,000 & ,000 & & ,007 \\
\hline & $\mathrm{N}$ & 397 & 397 & 397 & 397 & 397 & 397 & 397 & 397 & 397 \\
\hline \multirow{3}{*}{$\begin{array}{l}\text { CALIDAD DE } \\
\text { LOS PLATOS }\end{array}$} & \begin{tabular}{|l} 
Correlación de \\
Pearson
\end{tabular} & 098, & $1,000^{* *}$ & ,042 &,$- 315^{* *}$ &,$- 134 "$ &,- 086 &,- 011 &,$- 136^{\prime \prime}$ & 1 \\
\hline & Sig. (bilateral) & ,051 & 0,000 & ,399 & , 000 & ,007 & ,087 & 823 & 007, & \\
\hline & $\mathrm{N}$ & 397 & 397 & 397 & 397 & 397 & 397 & 397 & 397 & 397 \\
\hline
\end{tabular}

Fuente: Elaboración propia a partir del Trabajo de campo, 2019.

Se realiza una prueba aplicando la correlación de Pearson (Personal US, 2019) dice: "El valor del índice de correlación varía en el intervalo [-1,1], indicando el signo el sentido de la relación" para cada una de las variables investigadas con la muestra de 397 encuestados se observa (Sexo del encuestado y Nivel de estudios) hay una correlación del 0.098 y una significancia bilateral del 0.051 , existe una relación negativa en estas variables; en la relación con las otras variables el valor más alto es de $0.314^{* *}$ significativa en el nivel 0.021 a dos colas con una sig. Bilareral del 0.000 ; y la más baja de 0.010 con una sig. De 0.84 ; para las siguientes variables, se observa que en la preparación de platos el resultado 0.169 con una Sig 0.001 no existe una relación lineal ya que son dos variables independientes; en la variable de sexo y consigue materia prima los resultados de 0.206 y una sig. De 0.000 . 


\section{CONCLUSIONES}

Al analizar la satisfacción de los visitantes sobre la gastronomía típica y las características de la demanda de este tipo de establecimientos sirve para que los prestadores de servicios de restaurantes mejoren la oferta gastronómica acorde a la demanda, y de esta forma impacten de manera positiva en la experiencia de los visitantes cuando se realiza la visita.

Los resultados de esta investigación sugieren evidencia importante acerca del comportamiento de los visitantes en los restaurantes de gastronomía típica, por lo que estos locales son frecuentados en compañía de la familia, amigos y pareja. Acerca de las asociaciones entre variables socio-demográfica y variables de comportamiento, la variable quien toma la decisión a la hora de visitar un restaurante se encuentra influenciada por el género y la ocupación del visitante.

El atributo más importante en el servicio de los restaurantes de gastronomía típica es el sabor y la calidad de la comida, seguido del servicio y cortesía de los empleados y la variedad de los platos.

Se considera que uno de los factores más relevantes para la pérdida de la cultura gastronómica de Samborondón es la no preparación de platos por parte de la comunidad, en los restaurantes las preparaciones son en algunos de los casos simples y no bien elaboradas, pero a pesar de ello existe satisfacción en los platos ofertados.

El grado de satisfacción general medio es de 1.000 es decir que, el cual indica un nivel de satisfacción alta; datos que favorecen al potencial gastronómico del destino. Las variables que tienen mayor influencia sobre la satisfacción general del servicio analizadas a través de una correlación de Spearman son: el servicio y la cortesía de los empleados, el ambiente del establecimiento, la rapidez con la que lo atendieron y la variedad de los platos. 


\section{REFERENCIAS BIBLIOGRÁFICAS}

Ascanio, A. (2009). Rutas gastronómicas Chilenas: Una aproximación al tema. Pasos, 321-325.

Bel, C. y Arranz, A. (2011). El turismo y el desarrollo rural en los parques naturales. El caso del Parque Natural Sierra de Grazalema (Cádiz-Málaga) Spanish Journal of Rural Development, 1-22.

Bigné, E. y Font, X. (2000). Marketing de destinos turísticos. Análisis y estrategias de desarrollo. Esic Madrid.

Björk, P. \& Kauppinen-Räisänen, H. (2016). Local Food a Source for destination attraction. International Journal of Contemporary Hospitality Management, 177194.

Brida, J. G., y Aguirre, S. Z. (2010). Análisis del perfil y satisfacción de los pasajeros de cruceros que visitan el puerto de Cartagena de Indias. Turismo y Desarrollo Local.

Burns, A. C. (2000). Marketing research. Marketing research.

Chi, C. G. (2008). Examining the structural relationships of destination image, tourist satisfaction and destination loyalty: An integrated approach. Tourism Management, 624-636.

Fields, K. (2002). Demand for the gastronomy tourism product: motivational factors. Tourism and gastronomy, 36-50.

Flavián, C. (2011). Turismo gastronómico. Estrategias de marketing y experiencias de éxito. Prensas Universitarias de Zaragoza, Zaragoza.

Ignatov, E. (2006). Segmenting Canadian culinary tourist. Current Issues in Tourism, 235.

Jiménez, J.; López, T. \& González, F. (2016). Gastronomy and tourism: Profile and motivation of international tourism in the City of Córdoba, Spain. Journal of Culinary Science \& Technology, 347-362.

Kivela, J. J. (2009). Understanding travelers' experiences of gastronomy through etymology and narration. Journal of Hospitality \& Tourism Researc, 161-192. 
Kozak, M.; Bigné, E. \&. Andreu, L. (2004). Limitations of cross-cultural customer satisfaction research and recommending alternative methods. Journal of Quality Assurance in Hospitality \& Tourism, 37-59.

López, T.; Ribeiro, M. A.; Orgaz, F. y Marmolejo, J.A. (2015). El turismo en cabo verde: perfil y valoración del viajero. Estudios y Perspectivas de Turismo, 512-528.

M Mariani, M. A., y Sorio, A. (2010). Carne ovina, turismo e gastronomía: Pasos.

MINTUR. (2016). MINTUR. Obtenido de www.mintur.gob.ec

Oliveira, S. (2011). La gastronomía como atractivo turístico primario de un destino. Estudios y Perspectivas en Turismo, 738-725.

Oliver, R. L. (1980). A cognitive model of the antecedents and consequences of satisfaction decisions. Journal of Marketing Research XVII, 738-752.

OMT. (2016). Segundo Foro Mundial de Turismo gastronómico.

Personal US. (2019). https://personal.us.es/vararey/adatos2/correlacion.pdf. Obtenido de https://personal.us.es/vararey/adatos2/correlacion.pdf: https://personal.us.es/vararey/adatos2/correlacion.pdf

Petrick, J., \& Morais, D. D. (2001). An examination of the determinants of entertainment vacationers' intentions to revisit. Journal of Travel Research, 4148.

Rust, R.T. \&. Oliver, R. L. (1994). Service quality: New directions in theory and practice. Ssage.

Schlüter, R. y Gándara, J.M. (2008). Gastronomía y Turismo en Argentina. Pasos, 249268.

Yoon, Y. \&. Uysal, M. (2005). An examination of the effects of motivation and satisfaction on destination loyalty: a structural model. Tourism Managemen, 45-56. 